\title{
Numerical simulation on the mechanical characteristics of double-stranded DNA under axial stretching and lateral unzipping
}

\author{
C. A. Yuan ${ }^{\text {a) }}$ and G. Q. Zhang \\ Department of Precision and Microsystem Engineering, Delft University of Technology, \\ Delft, The Netherlands 2628 CD Delft \\ C. N. Han and K. N. Chiang \\ Advanced Microsystem Packaging and Nano-Mechanics Research Laboratory, Department of Power \\ Mechanical Engineering, National Tsing Hua University, HsinChu, Taiwan, Republic of China 30013 \\ Y. Cui \\ Department of Biomedical Engineering and Environmental Sciences, National Tsing Hua University, \\ Hsinchu, Taiwan, Republic of China 30013
}

(Received 3 October 2006; accepted 27 January 2007; published online 12 April 2007)

\begin{abstract}
The mechanical characteristics of the long-chain double-stranded DNA (dsDNA) molecule under the axial stretching and lateral unzipping are studied by the clustered atomistic-continuum method (CACM). The CACM consisted of the clustered atom method (CAM) and the atomistic-continuum method (ACM). The CAM treats the specific atomic group as the superatom, and the ACM describes the chemical binding energies between (super)atoms by virtual elements. The Newtonian based model of the dsDNA includes the superatoms of the backbones/base pairs and the virtual elements of the stacking energies/hydrogen bonds. The effective properties of the superatoms are numerically extracted from the single-stranded DNA experiments. Good agreements were achieved between the dsDNA numerical results and the single molecular experimental results. Via the simulation of stretching dsDNA, the mechanical responses, including the twisting of the backbone and variation of the elastic deformation energy and stacking energy, can be elucidated. Moreover, the predictive capability of the dsDNA CACM model is then examined. Furthermore, the dsDNA model with sequential information is subjected to the unzipping loading, and the in silico results reveal that the sliding of the backbones and the sequential dependent mechanical responses. (C) 2007 American Institute of Physics. [DOI: 10.1063/1.2715670]
\end{abstract}

\section{INTRODUCTION}

The single molecule manipulation techniques to measure the basic physical properties of single-stranded DNA (ssDNA) and double-stranded DNA (dsDNA) were developed rapidly during the last decade. This technique was successful in discovering the interaction between dsDNA and proteins/enzymes. ${ }^{1}$ Smith et $a l .{ }^{2}$ have stretched the bacteriophage lambda DNA which is immersed in the aqueous buffer to study the elastic properties of dsDNA by the dualbeam optical tweezers system. They discovered that stretching the freely rotating dsDNA has exhibited a sharp structural transition under roughly $65 \mathrm{pN}$ of tension. Leger et al. ${ }^{3}$ have also discovered that the S-form DNA occurs at the yielding point of DNA backbone while one stretch the freely rotating or underwound dsDNA. However, due to the limitation of the experimental resolution, the dynamic mechanical interaction of the chemical bonds, such as the $\pi$-stacking bonds and the hydrogen bonds, cannot be comprehensively elucidated by the molecular-level experiments. To overcome the limitations of the experiments, the molecular biology researchers require an essentially accurate theoretical or numerical model to analyze and predict the mechanical behavior of the dsDNA molecule. Benham ${ }^{4}$ had derived an

\footnotetext{
${ }^{a)}$ Author to whom correspondence should be addressed; electronic mail: c.a.yuan@tudelft.nl
}

analytical wormlike rod chain (WLRC) model for dsDNA mechanics and Sarkar et al. ${ }^{5}$ had improved its accuracy. These basic WLRC models can predict DNA's mechanical response under low stretching force. Zhou et al. ${ }^{6}$ proposed the Zhou-Zhang-Ou-Yang (ZZO) model, which considers the bending energy and the base pairs stacking energy of dsDNA. The ZZO model can describe B-S structural transition under high stretching force, but it is hard to represent the mechanical response under unzipping loading.

Consequently, we proposed a clustered atomisticcontinuum method (CACM), based on the Newtonian transient finite element method, to comprehensively investigate the mechanical behavior of dsDNA under external loading, as illustrated in Fig. 1. CACM combines the clustered atomistic method (CAM) and atomistic-continuum method (ACM). The CAM treats specific clustered atoms as superatoms with feasible characteristic properties. In dsDNA model, the nucleotides are treated as clustered elements, with characteristic properties extracted from the ssDNA experiments. The base-stacking energy and the hydrogen bond energy between the base pairs would be transformed into the two kinds of virtual finite elements by the ACM. The dsDNA mechanics behavior was implemented by the LS-DYNA3D transient finite element system within affordable CPU time. Compared with the molecule dynamics ${ }^{7}$ (MD) and the dissipative particle dynamics ${ }^{8}$ (DPD), the CACM could effi- 


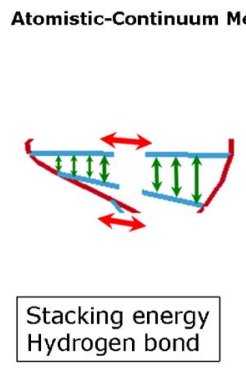

(a)

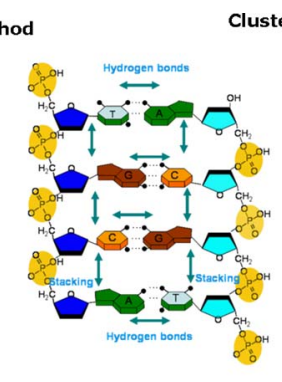

Clustered Atomistic Method

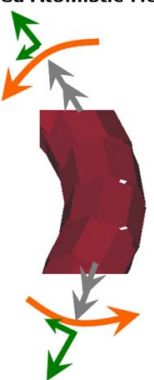

(b)
FIG. 1. The illustration of the dsDNA CACM model. (a) is the ACM model. The stacking energy and the hydrogen bond energy are treated as the virtual elements. (b) is the CAM model. The backbone and base pair of DNA are treated as clustered beam-type element (superatoms).

ciently enlarge the numerical time step ${ }^{9}$ and enhance the geometrical description of the clustered atomic groups (superatoms) with feasible equivalent mechanical properties.

\section{THEORY}

CACM is based on the transient finite element method, which simulates the mechanical behavior of a deformable body via minimizing the total potential energy. For a body $B$ composed of a set of material particles, we assume that the particle $X_{i}$ occupies the position $x_{i}$ at time $t_{n}$. Consequently, the equation $x_{i}=X_{i}+{ }^{t_{n}} u_{i}\left(X_{i}, t_{n}\right)$ holds, where ${ }^{t_{n}} u_{i}$ is the deformation of the position $x_{i}$ at time $t_{n}$. Additionally, the balance of the momentum of the body leads to the Cauchy equation of motion. $\partial \sigma_{j i} / \partial x_{j}+f_{i}=\rho \ddot{u}_{i}$ holds in body at each time $t_{n}$, where the $\sigma_{j i}$ denotes the Cauchy stress tensor, $f_{i}$ is the external force, and $\rho_{i}$ is the density. Therefore, one can generate the total potential energy of the body $B^{10}$ as

$$
\left.\delta \pi_{B}\right|_{t=t_{n}}=\left.\left(\delta P^{\mathrm{int}}+\delta P^{\mathrm{kin}}-\delta P^{\mathrm{ext}}\right)\right|_{t=t_{n}}
$$

where $P^{\text {int }}, P^{\mathrm{kin}}$, and $P^{\mathrm{ext}}$ represent internal, kinematical, and external loading energies. The $\delta$ represents the virtual quantities. The energy of external loading could be written as ${ }^{t_{n}} \delta P^{\mathrm{ext}}=\int_{A_{n}} \Delta T_{n} \delta u d A+\int_{V_{n}} \Delta f_{n} \delta u d V$, where $A_{n}$ and $V_{n}$ represent the area and the volume of body $B$ at time $t_{n}$, respectively. The $T_{n}$ and $f_{n}$ denote the surface traction and body force. Moreover, the internal energy and kinematical energy could be expressed as ${ }^{t_{n}} \delta P^{\mathrm{int}}=\int_{V_{n}}^{{ }^{t_{n}}} \sigma_{i j} \delta^{\delta^{n}} \varepsilon_{i j} d V$ and ${ }^{t_{n}} \delta P^{\mathrm{kin}}$ $=\int_{V_{n}} \delta u_{i} \rho^{t_{n}} \ddot{u}_{i} d V$, where the $\varepsilon_{i j}$ denotes the Green-Lagrange strain tensor.

To express the information of $t=i+1$ based on the information of $t=i$, the central difference method can be used. One could obtain the velocity of $t=i+\frac{1}{2}$ and the acceleration of $t=i$ as $\dot{u}_{i+(1 / 2)}=\left(u_{i+1}-u_{i}\right) / \Delta t$ and $\ddot{u}_{i}=\left[\dot{u}_{i+(1 / 2)}-\dot{u}_{i-(1 / 2)}\right] / \Delta t$, respectively. However, large $\Delta t$ can induce the numerical instability problem. In order to describe the phenomena of wave propagation, each element should be capable to represent at least one full wave. Therefore, the upper bound of the $\Delta t$ can be expressed as

$$
\Delta t=\alpha \Delta t_{\text {crit }}, \quad \Delta t_{\text {crit }}=\min \frac{l_{e}}{c_{e}},
$$

where $\alpha$ is a safety constant, $l_{e}$ is the characteristic length of the finite element, and $c_{e}$ is the wave speed.

The stacking energy occurs between the complementary base pairs of the dsDNA molecule. Electron cloud interactions $(\pi-\pi)$ between bases in the helical stacks contribute significantly to the stability of the double helix. The 12-6 Lennard-Jones (LJ) potential weak form with folding angle is used to describe the stacking energy as $U(r)$

$=(\varepsilon / r)\left[\left(\cos \rho_{0} / \cos \rho\right)^{12}-2\left(\cos \rho_{0} / \cos \rho\right)^{6}\right], \rho \geqslant 0$, where $\varepsilon$ is the base-stacking intensity, ${ }^{6} r_{0}$ is the backbone arc length between adjacent bases, and $\rho_{0}$ is the equilibrium distance between DNA dimer. ${ }^{6}$ Therefore, based on the CrottiEngesser theorem and geometrical assumptions, ${ }^{11}$ one can obtain the LJ potential force versus displacement,

$$
\begin{aligned}
f_{\mathrm{LJ}}=\frac{\partial U(\varphi)}{\partial l}= & \frac{12 A U_{0}}{l_{0}}\left(\frac{h_{0}+\Delta l \cos \varphi_{0}}{h_{0}+\Delta l}\right)^{7} \\
& \times\left[1-\left(\frac{h_{0}+\Delta l \cos \varphi_{0}}{h_{0}+\Delta l}\right)^{6}\right] \\
& \times\left[\frac{h_{0}\left(1-\cos \varphi_{0}\right)}{h_{0} \tan \varphi_{0}\left(h_{0}+\Delta l \cos \varphi_{0}\right)}\right],
\end{aligned}
$$

where $l_{0}, h_{0}$, and $\varphi_{0}$ represent the initial specific length, base pair height, and folding angle of the dsDNA, respectively. Equation (3) is represented as Fig. 2(a).

The hydrogen bond force is the interaction between complementary bases. Moreover, the GC base pair has three hydrogen bonds and AT has two. In the dsDNA CACM modeling, the three/two hydrogen bonds in GC/AT are replaced by only one virtual element, with the axial, shear, moment, and torsion stiffness as a function of the distance $\left(R_{0}\right)$ and the angle $\left(\theta_{i H j}\right)$ between the donor and the acceptor. The single hydrogen bond energy could be expressed as $E\left(R_{i}, \theta\right)$ $=\Sigma_{R_{i j}} A D_{0}\left[5\left(R_{0} / R_{i}\right)^{12}-6\left(R_{0} / R_{i}\right)^{10}\right] \cos ^{4} \theta_{i H j}$. Furthermore, we assume that the distances of the hydrogen bonds are the same along the dsDNA, the B-form DNA have the lowest hydrogen bond potential and D-H-A as a straight line, and the hydrogen atom always at the center of the hydrogen bond at initial state. Through the Crotti-Engesser theorem and geometrical assumptions, ${ }^{11}$ the reaction forces/bending moments could be expressed as

$$
F_{j}=\sum_{i} C_{(j, i)} \frac{\partial}{\partial x_{i}} E\left(R_{0}+d x_{i}, \theta_{0}+d \theta_{i}\right)
$$

where $i, j$, and $C_{(j, i)}$ represent the number of hydrogen bonds, different kinds of reaction forces/bending moments/torque, and weighting coefficients, respectively. Note that $x_{j}$ is the displacement along the hydrogen bond direction. The $\theta_{j}$ is the angle change between the donor and acceptor, and $\theta_{j}$ $=f_{j}(d x)$, where $f$ is the specific function with respect to $j$. Equation (4) is represented as Figs. 2(b)-2(e). 


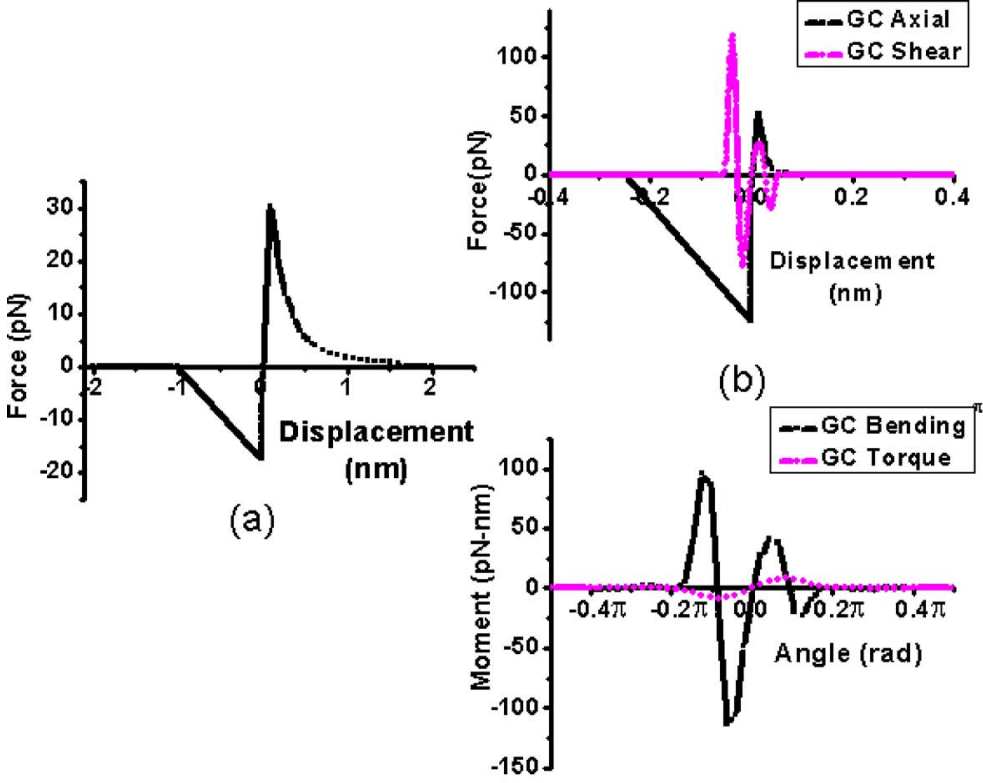

(d)

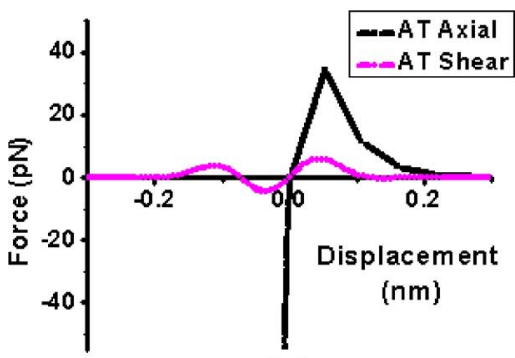

(c)

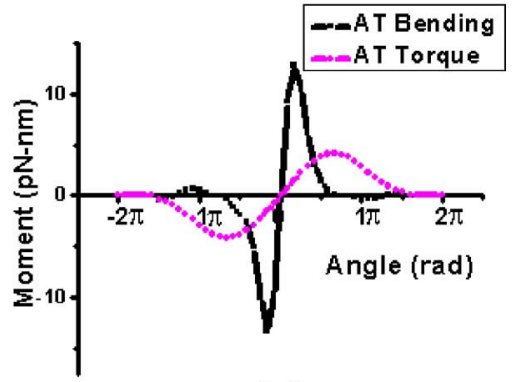

(e)

FIG. 2. The stiffness of the virtual elements. (a) is the virtual elements of the stacking energy with the folding angle equals $55^{\circ}$. (b) and (c) are the GC and AT axial and shear stiffness plots, respectively. (d) and (e) are the GC and AT bending and torque stiffness plots, respectively.

\section{MODELING}

The geometry of dsDNA is initially assumed as the double helix (B-form dsDNA) based on the helix function. As shown in Fig. 1(a), the force fields of the stacking and the hydrogen bond energies (Fig. 2) are added into the dsDNA model as the virtual elements. Moreover, two kinds of superatoms are used in the model. One is the combination of single phosphated based and five-carbon sugar, and the other represents the base pair, as indicated in Fig. 1(b). Notably, the beam-type finite element, with the reaction force of axial, shearing, moment, and torque, is applied to describe the behavior of the backbone and base pair superatoms.

The effective properties of these superatoms can be extracted from the experimental data of the ssDNA, because the base-stacking energy and hydrogen bond energy of ssDNA are neglectable. To simulate the experimental condition of stretching ssDNA, one end of the molecule was fixed and the other end was applied a prescribed displacement, as indicated in Fig. 3(a). For simplicity, the initial geometrical conformation of ssDNA is assumed as the B-form DNA and the super coiled behavior is neglected, as indicated in Fig. 3(b). The experimental results of ssDNA in phosphate buffer $^{2,12}$ were chosen as the benchmark to modify the effective elastic modulus of clustered backbone elements. Regarding to the numerical stability, the time step of the transient finite element computing is chosen as the smallest time step through the model at each different state, as shown in Eq. (2). The two peaks of simulation results which are shown in Fig. 3(c) occur while the local structural rotation of the ssDNA backbone is happening, ${ }^{12}$ but the experimental measurement cannot sense these responses because of the resolution limitation.

The experimental and simulated results are shown as the black and red lines in Fig. 3(c), respectively. The effective densities, elastic moduli, and diameters for the backbone/ base pair clustered element are $6.25 \times 10^{-3} / 5.00$ $\times 10^{-3}\left(\mathrm{~g} / \mathrm{nm}^{3}\right), 3940 / 15000\left(\mathrm{pN} / \mathrm{nm}^{2}\right)$, and 0.5/0.5 $(\mathrm{nm})$, respectively. Besides, the interactions of water and ions onto the dsDNA molecule are equivalent to the effective parameters of the superatoms, and this also causes why the value of the effective parameters are much larger than the realistic ones. The mechanical response of the dsDNA and ssDNA

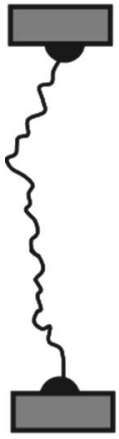

(a)

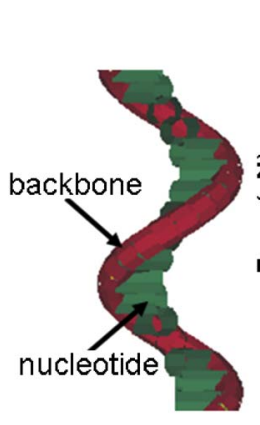

(b)

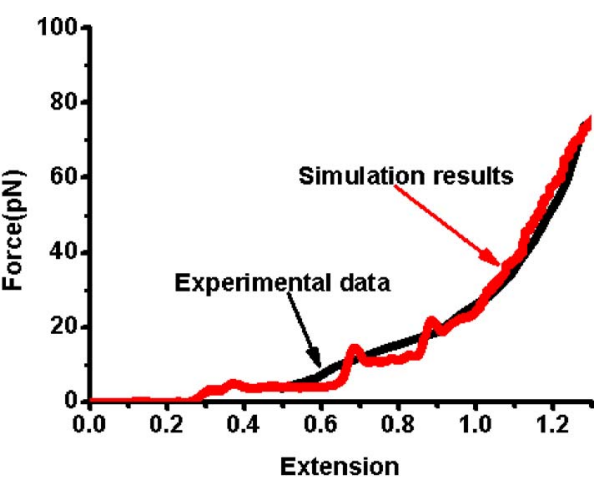

(c)
FIG. 3. The ssDNA model and the simulation results. (a) is the schematic illustration of ssDNA boundary conditions. (b) is segmental view of ssDNA model. (c) is the simulation results. 


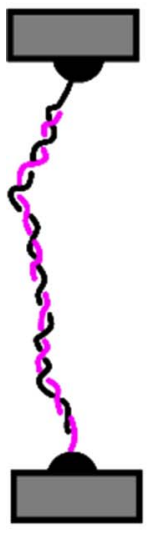

(a)

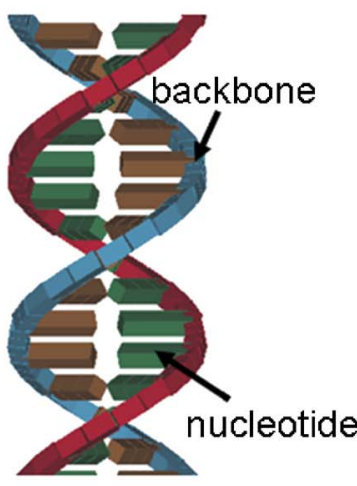

(b)

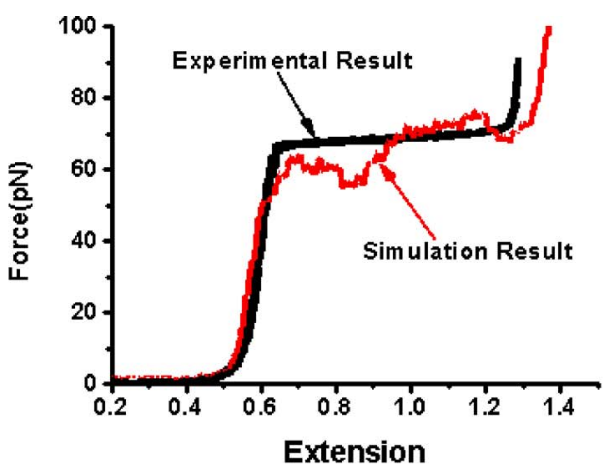

(c)

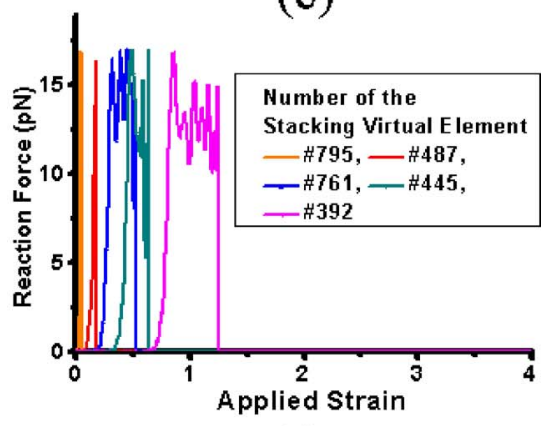

(d)
FIG. 4. The freely rotating dsDNA CACM model and the simulation results. (a) is the schematic illustration of boundary conditions. (b) is the segmental view of dsDNA CACM. (c) is the simulation result based on the dsDNA CACM model with 147 base pairs in (b). Note that The $x$ axis indicates the ratio of dsDNA deformation to the standard B-form DNA. (d) is the transient responses of the virtual elements of the stacking energies between the adjacent base pairs. The element 795 locates near where the external loading is applied, and also the following elements 487,761 , and 445. The element 392 locates near the fixed end of the model. model which is shown in the previous single molecular experimental results ${ }^{2,3,5,13}$ reveals that the molecules exhibit similar response under similar environments. Hence, under similar environments, the effective parameters of both ssDNA and dsDNA are assumed to be the same.

\section{STRETCHING dsDNA}

The dsDNA CACM model with 147 base pairs is established to simulate the dsDNA under the axial stretching loading, ${ }^{9}$ and both the freely rotating and both-strands-fixed boundary conditions are considered.

In the simulation of the freely rotating dsDNA, one end of specific strand is fixed and a prescribed displacement is applied to the other end of another strand, as illustrated in Fig. 4(a). The CACM dsDNA model with proper boundary and loading conditions is then solved by the transient finite element solver LS-DYNA ${ }^{14}$ based on Eqs. (1) and (2). The reacted forces, sensed by the bottom fixed point versus the external applied displacement, are shown in Fig. 4(c). The extension axis of Fig. 4(c) indicates the ratio of dsDNA deformation to the standard B-form DNA. A good agreement was achieved between the numerical and the experimental results. Note that we assume that the dsDNA molecule, which is longer than the persistence length $(\sim 147$ base pairs), will exhibit the similar mechanical response. The periodic boundary conditions are applied at the two ends of dsDNA model. Also, the information of dsDNA at low applied forces $(<1 \mathrm{pN})$ is ignored because the mechanical characteristics at large applied forces $(5 \sim 100 \mathrm{pN})$ are focused. Furthermore, the dsDNA model with 147 base pairs is used because of the issues around the computation time.
Through the simulation results, we can classify the mechanics of the dsDNA under stretching as three main stages as follows:

- (First stage) When external loading was first applied to the B-form DNA (B-DNA), the base pairs and hydrogen bond transfer the mechanical forces, bending moments, and torque between the two backbones. Both the backbone and the base-stacking virtual element inherited uniform reactant forces. Additionally, the torsional rigidity of the dsDNA backbones resisted the twisting of the complementary base pairs. Accordingly, the geometry of the dsDNA remained as B-DNA in this stage without any structural transition.

- (Seconds stage) As the external force was increased, the stacking reactant force between the adjacent base pairs also increased. When the distance between the adjacent base pairs exceeded the limitation, the basestacking virtual element would fail [Fig. 4(d)]. Moreover, the failure order of the stacking virtual elements will follow the geometrical location of these elements from loading end to the fixed end, as shown in Fig. 4(d). The B-S transition occurred at approximately $65 \mathrm{pN}$ of tension. Meanwhile, the torque of the dsDNA local structure overcame the backbone torsional rigidity and began untwisting the double helix. The untwisting started from the base pair nearest to where the external force was applied, and propagated along the remaining parts of the dsDNA.

- (Third stage) If the loading further increases, the dsDNA would be stretched as the ladder S-form DNA. In this stage, the base pairs and hydrogen bond transfer the mechanical forces/bending and moments/torque between the two backbones. The simulation depicted 


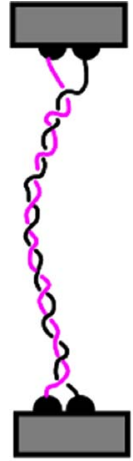

(a)

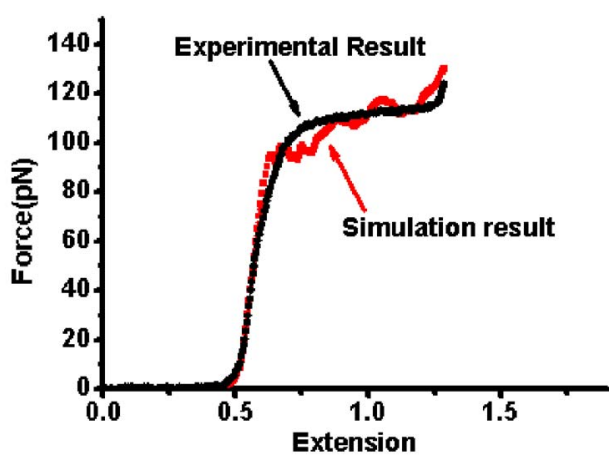

(b)
FIG. 5. The both-strands-fixed dsDNA CACM under the both-strands-fixed condition and the simulation result. (a) is the schematic illustration the boundary conditions. (b) is the simulation results.

that the backbones will inherit most of the reactant axial forces which are induced by the external loadings. Moreover, the base pairs will inherit most of the reactant torques which are induced by the structural transition (untwisting of the double helix at second stage).

Moreover, the boundary condition of the both strands fixed, as illustrated in Fig. 5(a) is then applied to the dsDNA model. Nodes at bottom of two strands are fixed in all degrees of freedom in the both-strands-fixed dsDNA simulation. Figure 5(b) shows the numerical results of the bothstrands-fixed dsDNA, where the extension is defined as the ratio of the deformed length to the standard B-form DNA, and the reaction force is extracted from the summation of the bottom fixed nodes. The experimental results indicated that the both-strands-fixed dsDNA exhibits a higher plateau about $110 \mathrm{pN}$ than the freely rotating one. Moreover, the simulation results coincide the experimental results with the maximum difference of the reaction force about $12.6 \%$ (at extension equals 1.33, far away from the plateau). Both the numerical simulation and the analytical prediction ${ }^{5,13}$ indicate that the B-S transition would occur during stretching the both-strands-fixed dsDNA, which is similar to the freely rotating one. Three structural transition stages could be apparently defined as the B-form dsDNA in stretching, B-S transition, and the S-form dsDNA in stretching.

Due to the fact that the initial configuration of the dsDNA model is assumed as B-DNA, both simulation results of freely rotating and both strands fixed exhibit the similar response at lower applied force. Moreover, both simulations [Figs. 4(c) and 5(b)] show differences between the computed and the measured forces near the beginning of second stage, and the possible reasons are as follows:

- These fluctuations can be filter by the long chain of dsDNA and the bead of the optical tweezers. Therefore, the experimental results did not show the fluctuations during the structural transition stage.

- The mechanical characteristics of backbone and base pairs, as well as the potential function of stacking energy and hydrogen bonds, are assumed the same throughout the simulation of first, second, and third

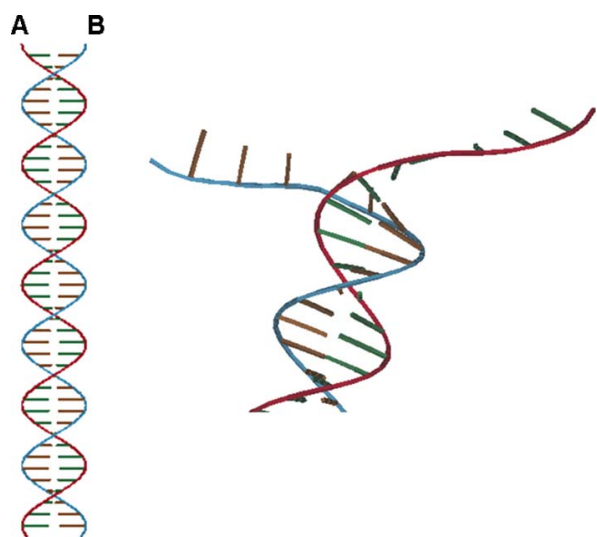

(a)

(b)

FIG. 6. The boundary and loading conditions of the unzipping dsDNA. (a) is the illustration plot of the boundary condition of dsDNA. Point $A$ is fixed, and point $B$ applied a prescribed motion toward to the right hand side. The reaction force could be sensed by point $A$, and the numerical results could be compared with the experimental results. (b) is the simulated deformation of the dsDNA molecule under the unzipping loadings.

stages. The local chemical/physical variations of dsDNA during the structural transition are not presented in the numerical model.

\section{UNZIPPING dsDNA}

Based on the aforementioned CACM dsDNA model, we studied the mechanical characteristics of the unzipping dsDNA. During gene expression, the base pairs are opened by the DNA enzyme or protein. Moreover, the opening process of the dsDNA is defined as the unzipping process in vitro. To simulate the unzipping dsDNA experiment, ${ }^{15-17}$ we implemented the boundary conditions as demonstrated in Fig. 6(a), and the expected deformation of the unzipping dsDNA is shown in Fig. 6(b). A prescribed motion, which is strictly proportional to the simulation time, is applied to the unzipping dsDNA CACM model. To understand the sequential dependent mechanical response, the GC-rich and AT-rich dsDNA CACM models are established. Moreover, the contact algorithm is applied onto the backbone to prevent the numerical penetration of the clustered elements.

The deformation shapes of GC-rich and AT-rich models are demonstrated in Table I. The maximum prescribed motion applied onto the model is approximately the length of the dsDNA model (42 base pairs). The sensed forces at the fixed point of the GC rich and AT rich are 24.85 and $12.8 \mathrm{pN}$, respectively, and these numerical results are quite similar to the experimental ones, which are $20 \pm 3$ and $9 \pm 3$ pN. $^{16}$

Figure 7(a) reveals the total energy of the GC-rich and AT-rich molecule, and the results depict that the GC-rich dsDNA can absorb more energy than the AT-rich one, because GC has three hydrogen bonds and AT has two. Additionally, [Fig. 7(a)] in the slope of the first few prescribed motion $(<0.5 \mathrm{~nm})$, two energy curves are overlapped because the external loadings are mainly absorbed by the backbones. Regarding to the stiffness of the virtual elements shown in Figs. 2(b)-2(e), the axial stiffness of GC and AT is 
TABLE I. The numerical results of the geometrical deformation of GC-rich and AT-rich dsDNA CACM model in unzipping loading (side view).

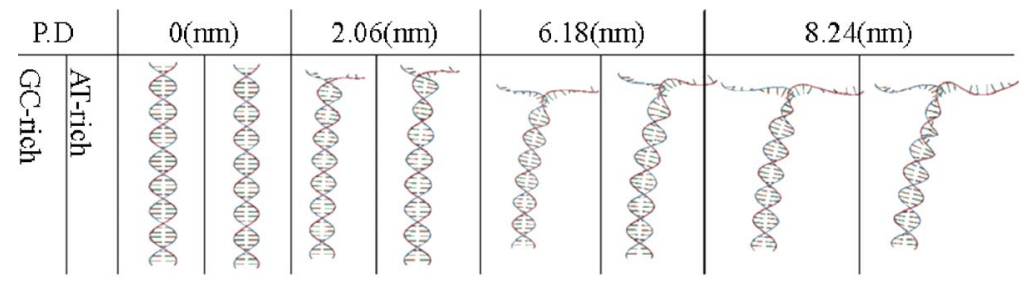

\begin{tabular}{|c|c|c|c|c|}
\hline P.D & \multicolumn{2}{|c|}{$12.36(\mathrm{~mm})$} & \multicolumn{2}{|c|}{$12.36(\mathrm{~nm})$} \\
\hline 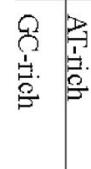 & $\frac{1}{9}$ & $\frac{1}{8}$ & $\frac{8}{8}$ & 5 \\
\hline
\end{tabular}

much higher than the others, and this also indicated that the failure of the hydrogen bond virtual elements can be shearing, moment, and torque. In the energy of the hydrogen bonds shown in Fig. 7(b), we discover that both the GC can reserve more energy than the AT. The hydrogen bond energy of the GC-rich model also depicts that the two plateaus occur at prescribed motion of approximately 4 and $12 \mathrm{~nm}$ [in the horizontal axis of Fig. 7(b)] because of the sliding of backbones. Owing to the fact that AT bond has lower stiffness

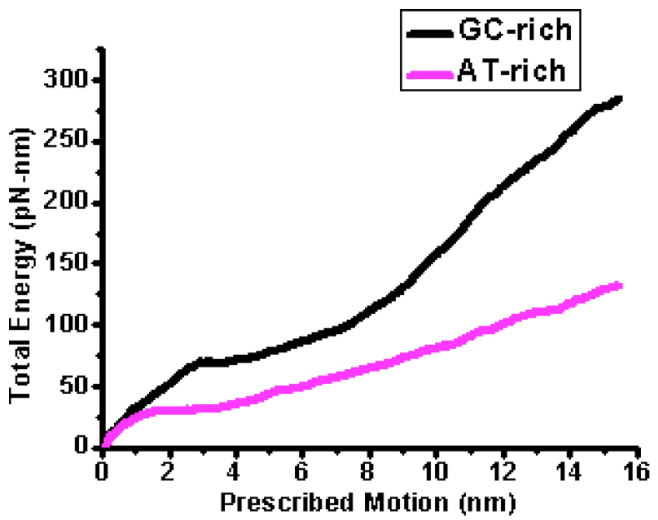

(a)

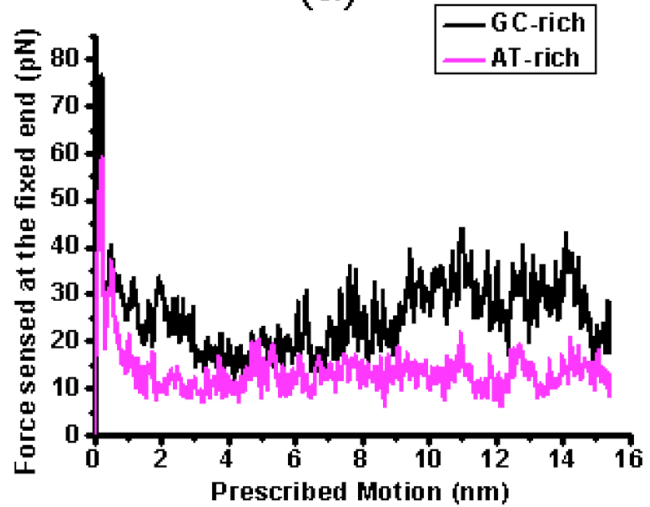

(c) than GC bond does the AT-rich model also depicts the two plateaus at prescribed motion of approximately 2 and $6 \mathrm{~nm}$ [shown in the horizontal axis of Fig. 7(b)]. The force responses which are obtained by the fixed node of GC-rich and AT-rich dsDNA is illustrated in Fig. 7(c). The simulation shows that the force AT-rich molecule is stable after the breaking of the hydrogen bonds. The contact energy plot [Fig. 7(d)] apparently depicts that both the two backbone of the GC-rich and AT-rich dsDNA would approach each other

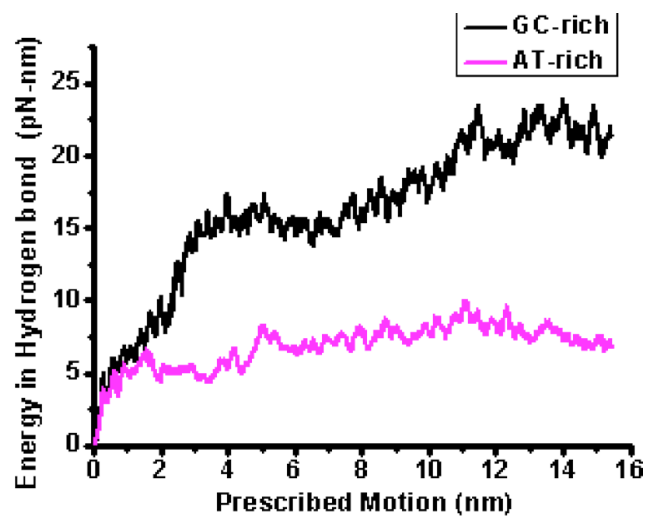

(b)

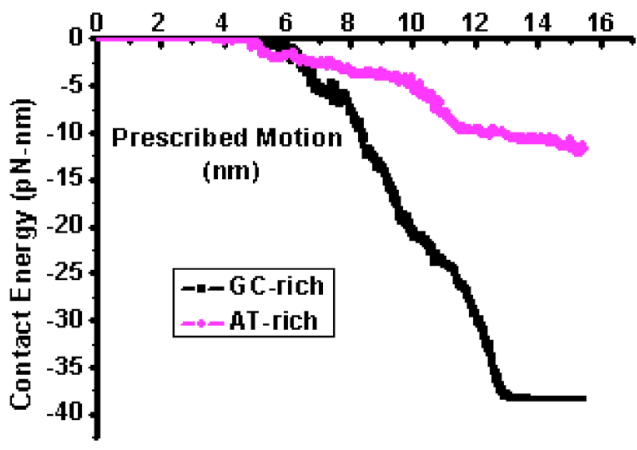

(d)

FIG. 7. The numerical results of unzipping GC-rich and AT-rich dsDNA. (a) is the total energy of the molecule plot. The diagram shows that the GC-rich molecule can restore more energy. The slope of the first few prescribed motion are the same because the external energy is absorbed by the backbone. (b) is the energy plot of the hydrogen bond. (c) Reaction force of the fixed end. (d) is the contact energy of the whole CACM model. After the contact occurs, the energy of hydrogen bond remains stable about 25 and $8.5 \mathrm{pN} \mathrm{nm}$, which is approximately 0.24 and $0.08 \mathrm{eV}$ for GC-rich and AT-rich molecule. 

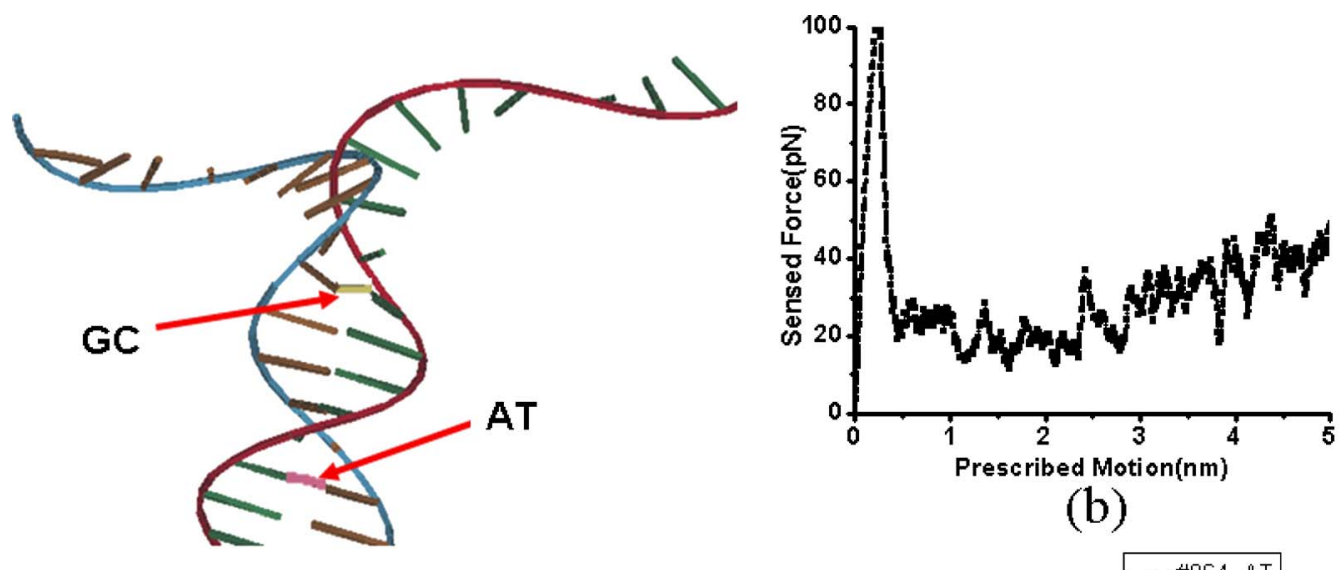

(b)

(a)

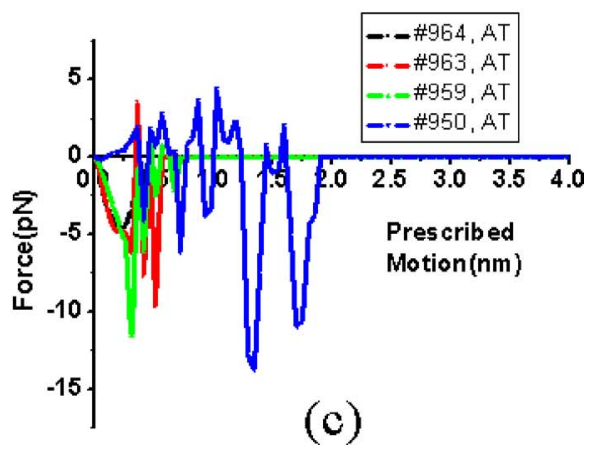

FIG. 8. The simulation results of dsDNA of bacteriophage lambda phage sequence. (a) is the opening geometrical plot. (b) is the reaction force (at the fixed end) vs the prescribed motion. (c) is the hydrogen bond response vs the prescribed motion.

and invoke the contact algorithm during the unzipping loading. The two backbones would not penetrate each other because of the Pauli's exclusive principle, however, the simulation depicted that two backbones would slide along each other. Moreover, the GC-rich dsDNA exhibits higher contact energy because the breaking of the GC bond favored the shearing direction more than the axial direction [as shown in Figs. 2(b) and 2(d)], and this phenomena would drive the GC-rich dsDNA to rotate and induce the contact of the two backbones. Mathematically speaking, the unzipping is one of the linking number releasing processes and the linking number is the summation of the twisting and writhing number. ${ }^{1}$ The ratio of the twisting and writhing depends on the structural rigidity of the molecule. For a short dsDNA fragment $(<20$ base pairs), the releasing of the writhing number will dominate the unzipping process. On the contrary, the linking number of the long dsDNA will be released by the twisting.

Based on the validated dsDNA CACM model, we modify the dsDNA CACM model and import the sequential information of the bacteriophage lambda phage. The sequence we used is CTTTTAAAAG TATTTATCGC TTTTGGGCGC TCCAGCGGCG G, which follows the opening direction. The sequence consists of $50 \% \mathrm{GC} / \mathrm{AT}$ pairs. The said model is subjected to the similar boundary and loading conditions as unzipping model. The simulated deformation shape of the sequential dsDNA CACM model under unzipping loading is illustrated in Fig. 8(a), where the pink bar represents the failure of the virtual AT hydrogen bond element. Based on the failure order of the hydrogen bond virtual elements, we discover that the AT pair fails prior to the GC pair (the dark yellow bar) after the backbones slide along each other. Therefore, the hydrogen bond between the base pairs would fail due to the fact that the combination of the axial force, shear force, bending moment, and the torque exceeds the critical criteria. The plot of the sensed force versus prescribed displacement is shown in Fig. 8(b). The peak of the force curves at the initial loading is because the loadings are suddenly exerted on the specific node in the numerical model. The information of the sensed force curve comprised the AT/GC pair mechanical response and the mechanical characteristic of the opened ssDNA backbone. Figure 8(c) shows the order of failure of the hydrogen bond as a function of the prescribed motion, and a sequential breaking of the AT bond can be observed. The force curve can be categorized as two plateaus which represent the AT-rich and GC-rich parts of $\lambda$-phage sequence as shown in Fig. 8(b).

\section{CONCLUSIONS}

The clustered atomistic-continuum method (CACM) is proposed to simulate the mechanical characteristics of the dsDNA under stretching and unzipping loading. The CACM consisted of the clustered atom method (CAM) which treats the specific atomic groups as the superatoms, and the atomistic-continuum method (ACM) which describes the chemical binding energy between (super)atoms. The experimental results of the ssDNA mechanical characteristics are used to extract the effective parameters of superatoms. The force fields of hydrogen bond and stacking energies are transformed into virtual elements. The simulation results of the freely twisting dsDNA demonstrate good agreements to the experimental results. Moreover, the structural transition of the dsDNA can be visualized and can be classified as three main stages, including B-form dsDNA in stretching, B-S 
structural transition, and S-form dsDNA in stretching. Furthermore, the both-strands-fixed dsDNA is analyzed and similar mechanical response is discovered.

The dsDNA CACM model is then applied to the unzipping loading to study the mechanical difference between sequential information. For GC-rich and AT-rich sequences, the sensed opening force of dsDNA obtained by the numerical simulations are 24.8 and $12.8 \mathrm{pN}$ (in average), where the experimental results are $(20 \pm 3)$ and $(9 \pm 3) \mathrm{pN}$, respectively. Moreover, the sliding of the backbones during the unzipping loading is demonstrated by the simulation. Afterwards, the mechanical response of dsDNA CACM model of the bacteriophage lambda phage sequence is then studied. The simulation reveals that the sequential difference can induce the different mechanical characteristics. Therefore, the dsDNA CACM model has the capability to simulate the dsDNA under various loading/boundary conditions in silico.

\section{ACKNOWLEDGMENTS}

The authors are grateful to Dr. J. Marko, Dr. S. B. Smith, and Dr. C. Bustamante. Also, the authors thank Dr. A. Sarkar for valuable discussions on single molecular experiment. Moreover, the authors thank Dr. S. Atluri, Dr. I. C. Hsu, and Dr. C. S. Cheng for the valuable suggestions. One of the authors (C.A.Y.) thanks Dr. J. Day and Dr. C. Tsay for discussions on numerical simulation technique.
${ }^{1}$ C. Bustamante, Z. Bryant, and S. B. Smith, Nature (London) 421, 423 (2003).

${ }^{2}$ S. Smith, Y. Cui, and C. Bustamante, Science 271, 795 (1996).

${ }^{3}$ J. F. Leger, G. Romano, A. Sarkar, J. Robert, L. Bourdieu, D. Chatenay, and J. F. Marko, Phys. Rev. Lett. 83, 1066 (1999).

${ }^{4}$ C. Benham, Phys. Rev. A 39, 2582 (1989).

${ }^{5}$ A. Sarkar, J. F. Leger, D. Chatenay, and J. F. Marko, Phys. Rev. E 63, 51903 (2001).

${ }^{6}$ H. Zhou, Y. Zhang, and Z. Ou-Yang, Phys. Rev. E 62, 1045 (2000).

${ }^{7}$ M. Rieth, Nano-engineering in Science and Technology, 1st ed. (World Scientific, New Jersey, 2003).

${ }^{8}$ L. Jupp, T. Kawakatsu, and X. F. Yuan, J. Chem. Phys. 119, 6361 (2003).

${ }^{9}$ K. N. Chiang, C. A. Yuan, C. N. Han, C. Y. Chou, and Y. Cui, Appl. Phys. Lett. 88, 023902 (2006).

${ }^{10}$ R. D. Cook, D. S. Malkus, and M. E. Plesha, Concepts and Application of Finite Element Analysis, 3rd ed. (Wiley, New York, 1989).

${ }^{11}$ C. A. Yuan, C. N. Han, and K. N. Chiang, Proceedings of the NSTI Nanotechnology Conference, Vol. 2, Chapter 8, pp. 561-565, Anaheim, CA, May 2005.

${ }^{12}$ C. N. Han, C. Y. Chou, C. J. Wu, and K. N. Chiang, Proceedings of the NSTI Nanotechnology Conference, Vol. 2, Chapter 4, pp. 321-324, Boston, May 2006.

${ }^{13}$ F. Allemand, D. Bensimon, R. Lavery, and V. Coqustte, Proc. Natl. Acad. Sci. U.S.A. 95, 14152 (1998).

${ }^{14}$ J. O. Hallquist, LS-DYNA Theory Manual (Livermore Software Technology, Livermore, CA, 1998).

${ }^{15}$ M. D. Wang, M. J. Schnitzer, H. Yin, R. Landick, J. Gelles, and S. M. Block, Science 282, 902 (1998).

${ }^{16}$ M. Rief, H. Clausen-Schaumann, and H. Gaub, Nat. Struct. Biol. 6, 346 (1999).

${ }^{17}$ U. Bockelmann, Ph. Thomen, B. Essevaz-Roulet, V. Viasnoff, and F. Heslot, Biophys. J. 82, 1537 (2002). 